\title{
A Simulation Study on the Growth of Oviduct Mucosa Cells in the Uterine Cavity Microenvironment
}

\author{
Cheng-Rong $\mathrm{Wu}^{\prime}$ \\ Hua-Lei Cai ${ }^{2}$ \\ Di-Xian Cai ${ }^{3}$ \\ Tao-Lan $\mathrm{Li}^{1}$ \\ Rong Shen ${ }^{2}$ \\ Dan $\mathrm{Zi}^{2}$
}

'Department of Obstetrics and Gynecology, Guizhou Medical University, Guiyang, 550004, People's Republic of China; ${ }^{2}$ Department of Obstetrics and Gynecology, The Affiliated Hospital of Guizhou Medical University, Guiyang, 550004, People's Republic of China; ${ }^{3}$ Department of Psychology, Guizhou Medical University, Guiyang, 550004, People's Republic of China
Correspondence: Hua-Lei Cai Department of Obstetrics and Gynecology, The Affiliated Hospital of Guizhou Medical University, No. 28 of Guiyi Street, Yunyan District, Guiyang, 550004, People's Republic of China

Tel $+86 I 8 I 98 I 32660$

Fax +86 85I-86855II9

Email caihualei_dr@I63.com
Objective: The growth of oviduct mucosa in the uterine cavity was observed by co-culture of oviduct mucosa cells and endometrial cells in different proportions to study the possibility and function of the growth of oviduct mucosa in the uterine cavity.

Methods: The extracted cells were identified by immunofluorescence with cytokeratins 19 (CK19) and vimentin. A Cell Counting Kit-8 (CCK8) experiment, cell decidualization induction, and HE staining were performed after the co-culture of two kinds of cells in different proportions.

Results: 1) The cells could grow normally when the two cells were co-cultured indirectly. 2) A CCK8 test of oviduct mucosa cells showed that the growth rate of each group was similar after the indirect co-culture of two kinds of cells in different proportions, which was in line with the growth law of normal cells. 3) Immunofluorescence identification of the cells showed that most of the two kinds of cells in the second passage were CK19 positive and were epithelial cells, while most of the cells in the fifth passage expressed positive vimentin antibody and were stroma cells. 4) After cell decidualization induction, the cell morphology of each group showed deciduation-like changes. 5) After decidualization, the cell morphology of each group was similar after HE staining. Conclusion: Oviduct mucosa cells can grow normally in the uterine environment. In the uterine environment with different degrees of endometrial loss, the growth rate of oviduct mucosa cells is not inhibited. Its morphology does not change, and it can undergo decidualization in vitro.

Keywords: oviduct mucosa, endometrium, co-culture, endometrial receptivity, intrauterine adhesion

\section{Introduction}

At present, in reproductive medicine, endometrial loss or insufficient endometrial thickness has become one of the main problems that affect the successful implantation and normal growth of embryos. Therapeutic methods used to promote endometrial regeneration at home and abroad include exogenous estrogen, low-dose aspirin, sildenafil citrate, pentoxifylline, vitamin E, L-arginine, cytokines, electroacupuncture, and biofeedback therapy. Although these treatments claim to improve the implantation and pregnancy rates during the assisted reproductive technology cycle, from the perspective of evidencebased medicine, various methods for treating thin endometrium seem to be useless and inefficient, and patients with thin or even deficient endometrium are not likely to attempt pregnancy through assisted reproduction. ${ }^{1}$ Therefore, researchers have been exploring innovative interventions for the treatment of thin endometrium and endometrium loss. 
New measures to treat thin endometrium and endometrium deletion are urgently needed in the clinical setting. According to the existing knowledge, oviduct mucosa and endometrium have many similarities in tissue structure and function. In terms of tissue structure, they share the homology of embryo origin. The cellular components of mucosa and intima are composed of epithelial and mesenchymal components, and the epithelial cells are mainly composed of ciliary and secretory cells, with similar cell morphology but different cell proportion. In terms of function, both of these two mucous membranes undergo morphological, physiological, and biochemical changes, along with changes in ovarian hormones, during the menstrual cycle. These changes have many similarities and are the necessary basis for the formation and growth of early embryos. ${ }^{2}$ For example, oviduct and uterine fluids contain growth factors, cytokines, proteins, metabolites, etc., which provide nutritional support and immune protection for the normal growth and development of early embryos., Oviduct mucosa and endometrium have the same "implantation window". ${ }^{-7}$ Studies have confirmed that the chromosome analysis of trophoblast cells of embryos from ectopic salpingocyesis is not significantly different from that of normal intrauterine pregnancy, so it is considered that salpingocyesis is the implantation of normal embryos in oviduct mucosa. ${ }^{8}$ Based on that, we hypothesized that placing oviduct mucosa into the uterine cavity to perform the endometrium function might be a way to treat thin endometrium and endometrium loss. Therefore, we co-cultured primary oviduct mucosa cells and endometrial cells to understand the growth of the two types of cells during co-culture and to simulate different levels of intimal loss, to observe the growth and status of oviduct mucosa cells cultured under the condition of endometrium deletion, and to lay a foundation for the possibility of replacing endometrium with oviduct mucosa in the treatment of intrauterine adhesions (IUA).

\section{Materials and Methods}

\section{Subjects}

At the Affiliated Hospital of Guizhou Medical University from November 2019 to December 2020, oviduct mucosa and endometrium were collected from 22 gynecological patients who needed to have their endometrium, oviduct, and uterus removed due to infertility, myoma of the uterus, adenomyosis, CIN III, and other diseases. The average age of the patients was 39.4 years old, and most of these patients were in the middle of their menstrual cycle (late proliferation). The postoperative pathological examination proved that there were no malignant pathological changes in the oviduct and endometrium, and they had not taken steroid hormones three months before surgery.

The 22 participants were selected for this study for the following reasons: 1 . All of these 22 patients need to have their fallopian tubes removed due to their condition, and specimens of fallopian tubes can be taken for this experiment; 2 . These 22 patients need to have endometrial biopsy at the same time, and there is residual endometrial tissue available for this experiment; 3 . Carry out the experiment in the article, 22 patients' specimens are just enough to repeat this experiment 3 times; 4. These 22 patients are all nonmalignant tumor diseases, and the pathological examinations of fallopian tube mucosa and endometrial are normal, which is suitable for this Research; 5. These patients are all at childbearing age, have regular menstrual cycles, and have no endocrine diseases.

All tissue samples were collected with informed consent signed by the patients. The Ethics Committee of the Affiliated Hospital of Guizhou Medical University approved this study.

\section{Extraction of Endometrium and Oviduct Mucosa Primary Cells}

After the uterus and oviduct were removed from the body, the endometrium was taken immediately, and oviduct mucosa was separated and collected in sterile specimen bottles containing normal saline. The samples were stored at $4^{\circ} \mathrm{C}$ and sent to the laboratory within $1 \mathrm{~h}$. The specimens were removed and placed in a Petri dish with a diameter of $3.5 \mathrm{~cm}$. The surface red blood cells were washed with phosphate buffer solution (PBS) three times, and the tissues were cut into small pieces less than $1 \mathrm{~mm}$. The tissue pieces were moved to a $15 \mathrm{~mL}$ centrifuge tube with a pipette, washed once with PBS solution, and centrifuged for $5 \mathrm{~min}$ at $1,200 \mathrm{r} / \mathrm{min}$, and the supernatant was discarded. A $0.1 \mathrm{mg} / \mathrm{mL}$ of type I collagenase (C0103, Sigma) was added for digestion and placed in a $37^{\circ} \mathrm{C}$ constant temperature water bath, during which the digestion was vibrated every $10 \mathrm{~min}$. After $90 \mathrm{~min}$ of digestion, Dulbecco's Modified Eagle Media: Nutrient Mixture F-12 (DMEM/F12) was added to terminate digestion. The endometrium and oviduct mucosal suspensions were centrifuged at 1,200 $\mathrm{r} / \mathrm{min}$ for $5 \mathrm{~min}$ to remove the supernatant and obtain the sediment. A $10 \%$ fetal bovine serum (super fetal bovine serum [South America], 04-001-1ACS, BI) was added to the two sediments respectively, blown and mixed well, and inoculated in a cell culture flask. The endometrium and oviduct mucosal cell 
culture bottles were incubated in a $\mathrm{CO}^{2}$ incubator at $37^{\circ} \mathrm{C}$ for $24 \mathrm{~h}$ to observe cell attachment and growth. If the cells were attached and growing well, the solution was changed every 48 $\mathrm{h}$, and the cell morphology was observed with an inverted microscope.

\section{Cells Passage}

When the bottom of the culture bottle was about 80 90\% full of cells, the original medium was discarded and washed twice with PBS, $0.25 \%$ pancreatic enzymes were added just above the bottom of the bottle, and the culture bottle was placed in an incubator with $5 \% \mathrm{CO}^{2}$ at $37^{\circ} \mathrm{C}$ for digestion for 1-2 min. After the cells were suspended, digestion was stopped with DMEM-F12, which was 4-5 times the volume of the pancreatic enzymes, and centrifuged at 1,200 r/min for $5 \mathrm{~min}$. The supernatant was removed, and the cell sediment was retained. A $10 \%$ fetal bovine serum complete medium was added to the cell sediment, thoroughly beaten and mixed, and a 105 cell suspension was inoculated in the culture flask. A $10 \%$ fetal bovine serum complete medium was supplemented in the culture flask to $6 \mathrm{~mL}$ and placed in an incubator of $5 \% \mathrm{CO}^{2}$ at $37^{\circ} \mathrm{C}$.

\section{Cell Identification}

The second generation's endometrial and oviduct mucosa cells, which were in a logarithmic growth phase and good growth state, were selected. The sterile slides were placed into a 12-well Petri dish, and the slides were taken out at a rate of $4 \times 10^{4}$ cells per well when the cells had grown to cover about $80-90 \%$ of the slides. The slides were then fixed with $4 \%$ formaldehyde for $15 \mathrm{~min}$ and $0.5 \%$ Triton X-100 membrane broken for $20 \mathrm{~min}$, sealed with $10 \%$ normal goat serum PBS for $30 \mathrm{~min}$, rabbit anticytokeratin (Cytokeratin 19 Antibody, 10712-1-AP, ProteinTech, 1:300), and PBS (blank control) and were incubated overnight in the dark at $4^{\circ} \mathrm{C}$. The first antibody was recovered, washed with PBS three times, and then incubated with goat anti-rabbit green fluorescent second antibody (488, SA00006-2, ProteinTech, 1:200) for 1 h. Then it was washed with PBS three times for $5 \mathrm{~min}$ each time, a small amount of DAPI staining was added for 5 min, dried, sealed with an anti-fluorescent quenching agent, and preserved under a fluorescence microscope. When the endometrial cells and oviduct mucosa cells were subcultured to the fifth generation, the cells with good growth status and logarithmic growth phase were identified with Vimentin antibody (10366-1-AP, ProteinTech, 1:300). The secondary antibody was marked by red fluorescence Alexa Fluor 594-conjugated Goat Anti-rabbit IgG (H+L) (SA00006-4, ProteinTech, 1:200). The steps were the same as above, photographed and saved by fluorescence microscope.

\section{Grouping, Cell Co-Culture, and Decidualization}

The groups were as follows:

A. oviduct mucosal cells (control group);

B. endometrial cells (upper chamber) + oviduct mucosal cells (lower chamber), cell ratio of the upper chamber: lower chamber was 1:1 (experimental group 1);

endometrial cells (upper chamber) + oviduct mucosal cells (lower chamber), cell ratio of the upper chamber: lower chamber was 0.5:1 (experimental group 2);

endometrial cells (upper chamber) + oviduct mucosal cells (lower chamber), cell ratio of the upper chamber: lower chamber was 0.1:1 (experimental group 3).

Cell co-culture and decidualization: the fifth-generation oviduct mucosa cells in the logarithmic growth phase and good growth state were selected, and $1.8 \times 10^{5}$ cells/well were inserted into the six-well plate to grow simultaneously in the lower chamber. At the same time, the fifth generation of endometrial cells, which were in a logarithmic growth stage and good growth state, were collected and inserted into the upper chamber with $1.8 \times 10^{5}, 9 \times 10^{4}$, and $1.8 \times 10^{4}$ cells per well.

Endometrial cells and oviduct mucosal cells were cocultured with $2 \%$ fetal bovine serum after the cells adhered to the wall. After $48 \mathrm{~h}$ of culture, change to the medium for inducing cell decidualization (ingredients of the medium for inducing cell decidualization: cell culture medium with $2 \%$ fetal bovine serum, $1 \mu \mathrm{m}$ progestational hormone [Progesterone, V900699, Sigma], 10 nM 17- $\beta$ estradiol [ $\beta$ Estradiol, E110145, Aladdin], $0.5 \mathrm{mM}$ 8-Br-cAMP [8-Bromoadenosine 3',5'-cyclic monophosphate, B5386, Sigma]) was continuously cultured for $96 \mathrm{~h}$; cell growth and cell changes after decidualization were observed under an inverted microscope.

\section{CCK8 Cell Proliferation Assay (Growth Curve Was Drawn)}

The third generation of oviduct mucosal cells, which were in the logarithmic growth phase and good growth state, were added to the lower compartment of a 24-well plate at a rate of $5 \times 10^{3}$ cells per hole. At the same time, the third generation of endometrial cells in the logarithmic growth 
phase and good growth state were collected and placed into the upper chamber with $5 \times 10^{3}, 2.5 \times 10^{3}, 5 \times 10^{2}$ / well plates. Endometrial cells were cultured with oviduct mucosa cells after the cells adhered to the wall. The same group was incubated for seven consecutive days. The tunnel chamber was removed daily, and the medium was sucked out of the 24-well plate, and $500 \mu \mathrm{L}$ fresh medium was added for each well, and $50 \mu \mathrm{L}$ CCK 8 was added to each well and cultured at $37^{\circ} \mathrm{C}$ for $2 \mathrm{~h}$. Each well absorbed $100 \mu \mathrm{L}$ of the medium and was added to the 96-well plate. Each well had three replicas. The absorbance value of each well was measured by OD (optical density) at $450 \mathrm{~nm}$ by the enzyme calibration.

\section{HE Staining}

The cell slides were placed in the lower and upper compartments of the 12-well plate co-cultured in the compartment. The fifth generation of oviduct mucosal cells in the logarithmic growth phase and good growth state were taken and inserted into the lower compartment slides of the 12-well plate co-cultured at a rate of $4 \times 10^{4}$ cells per hole. At the same time, endometrial cells of the fifth generation, which were in a logarithmic growth phase and good growth state, were collected and inserted into 12-well plates to grow the upper chamber. Endometrial cells were cultured with oviduct mucosal cells after the cells were attached to the slit. The grouping method was the same as above. After decidualization, the four groups of cell slides were soaked with PBS three times, 3 min each. The slide was fixed with 4\% paraformaldehyde for $15 \mathrm{~min}$, and the slide was soaked by PBS three times, 3 min each. The climbing film was dripping with Mayer's hematoxylin (with a clean staining background, without differentiation), dyed for $10 \mathrm{~min}$, and washed and soaked in tap water to return to blue. Next, the climbing film was dripping with $1 \%$ water-soluble eosin dye for $5 \mathrm{~min}$, washed and soaked in tap water for $30 \mathrm{~s}, 95 \%$ alcohol I for $30 \mathrm{~s}, 95 \%$ alcohol II for $1 \mathrm{~min}$, absolute ethyl alcohol I for $5 \mathrm{~min}$, absolute ethyl alcohol II for 5 min, xylene I for $5 \mathrm{~min}$, and xylene II for $5 \mathrm{~min}$, and sealed with neutral balsam after air drying; this was then examined with a microscope.

\section{Statistical Analysis}

Normally distributed variables were expressed as mean \pm s.d. For repeated measurement data, repeated measurement ANOVA was used. P-values less than 0.05 were considered statistically significant. Analyses were carried out using the GraphPad (version 7.0; GraphPad Software, Inc.) and SPSS software (version 22.0; IBM Corp).

\section{Results \\ Morphology of Endometrial Cells and Oviduct Mucosal Cells}

The primary cultured cells were observed under an inverted microscope. Endometrial cells were completely adherent within $24 \mathrm{~h}$, and oviduct mucosa cells adherent after $48 \mathrm{~h}$. Both cells were polygonal, tadpole, and spindle types in the primary culture and early passage. The nucleus was centered and round, and the cells gathered and grew like islands. Most of the cells began to change in morphology after passage to the third generation and gradually appeared as long fusiform. By the fifth generation, most cells grew into fusiform, similar to the fibrous cells (Figure 1).

\section{Cell Identification}

Immunofluorescence identification of epithelial marker keratin antibody was performed on endometrial and oviduct mucosa cells passed to the second generation. The results showed that $80-90 \%$ of the cytoplasm of endometrial and oviduct mucosa cells were stained with green fluorescence after passage to the second generation, showing positive cytokeratin antibodies. Microscopically, the cells were polygonal, spindle and spindle. Immunofluorescence identification of interstitial cell marker vimentin antibody was performed on endometrial and oviduct mucosa cells passed to the fifth generation. Eighty percent of the cytoplasm of endometrial and oviduct mucosa cells were stained with red fluorescence under a microscope. It showed a positive cell vimentin antibody, and the cells were fusiform and polygonal under the microscope. According to the results of cell identification, the majority of the two kinds of cells were still epithelial cells before the second passage, and the epithelial cells gradually transformed into mesenchymal cells when the passage continued. After the fifth passage, the cultured cells were mainly stromal (Figure 2).

\section{Growth Curve}

According to the same grouping method mentioned above, the growth curve was drawn after seven days of continuous measurement of endometrial cells and oviduct mucosa cells after co-culture. It can be seen that the growth rate of oviduct mucosa cells, when growing alone, was similar to that of endometrial cells in different proportions when co-cultured, 


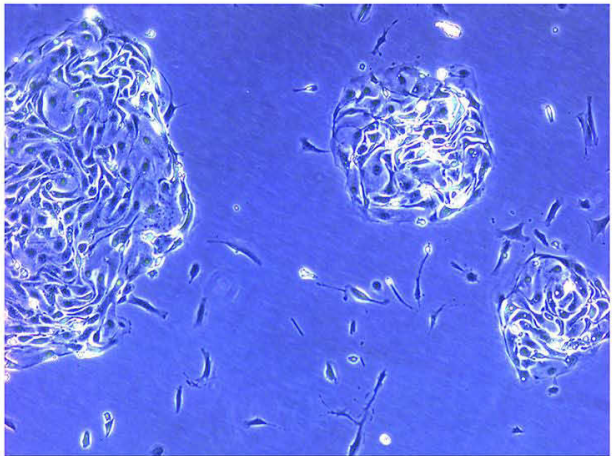

A

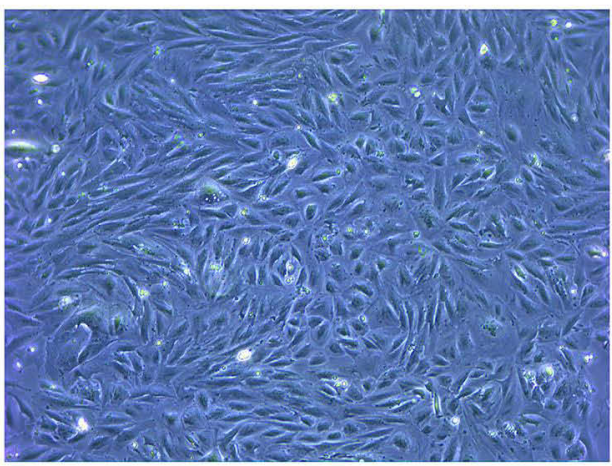

C

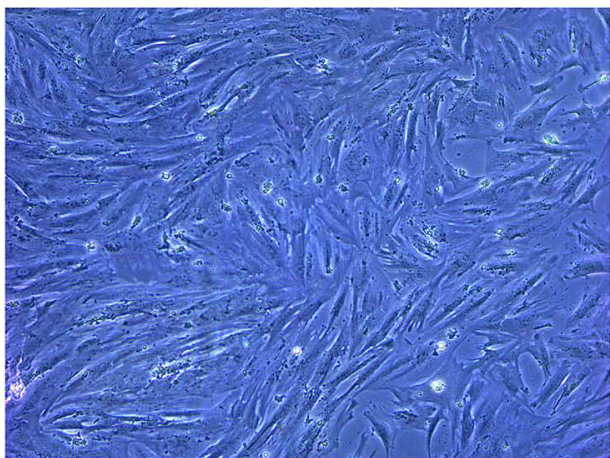

E

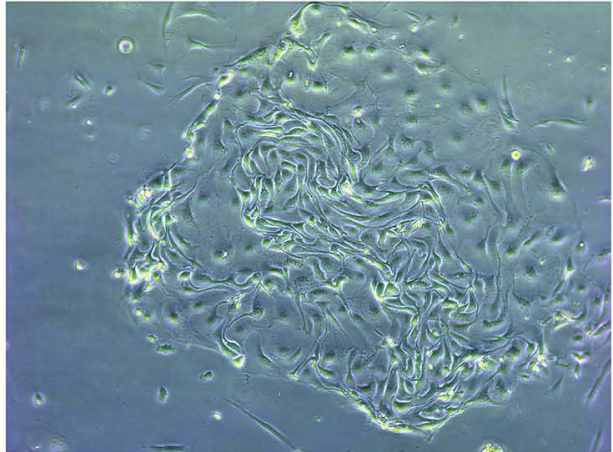

B

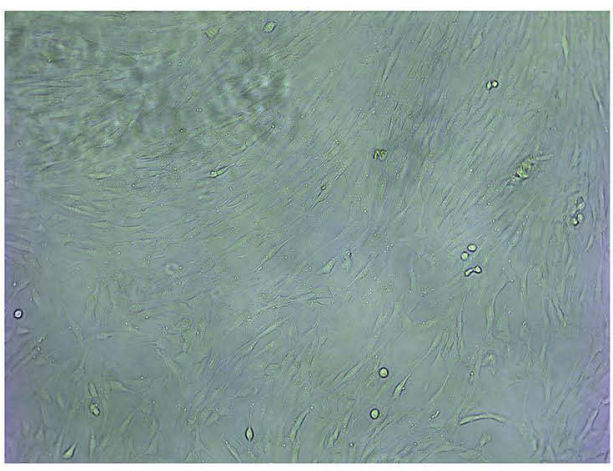

D

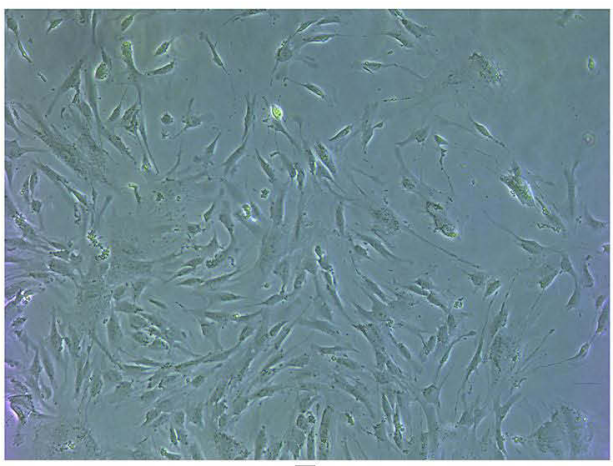

F

Figure I The primary cultured cells were observed under an inverted microscope. Endometrial cells were completely adherent within 24 hours and oviduct mucosa cells adherent after 48 hours. Both cells were polygonal, tadpole and spindle type in primary culture and early passage. The nucleus was centered and round, and the cells gathered and grew like islands. Most of the cells began to change in morphology after passage to the third generation, and gradually appeared long fusiform. By the fifth generation, most of the cells grew into fusiform, similar to fibrous cells. (A) Primary endometrial cells; (B) primary oviduct mucosal cells; (C) the third generation of endometrial cells; (D) the third generation of oviduct mucosal cells; (E) the fifth generation of endometrial cells; $(\mathbf{F})$ the fifth generation of oviduct mucosal cells.

without a significant statistical difference $(\mathrm{P}>0.05)$. The growth curve was of the "S" type, which means they experienced three stages: slow growth stage, logarithmic growth stage, and plateau stage (Figure 3).

\section{Decidualization Treatment}

According to the same grouping method mentioned above, endometrial cells were co-cultured with oviduct mucosa epithelial cells and treated with decidualization. After 48 $\mathrm{h}$ of culture, oviduct mucosa cells induced by decidualization began to show decidual-like changes, with large and round cell volumes, rich and transparent cytoplasm, and blurred cell boundaries (Figure 4).

\section{HE Staining}

The cells in each group were treated with decidualization by HE staining, and the cytoplasm was stained eosin, and the nucleus was blue. When oviduct mucosa cells were cultured alone and co-cultured with endometrial cells of different proportions, the cell morphology was unchanged, 


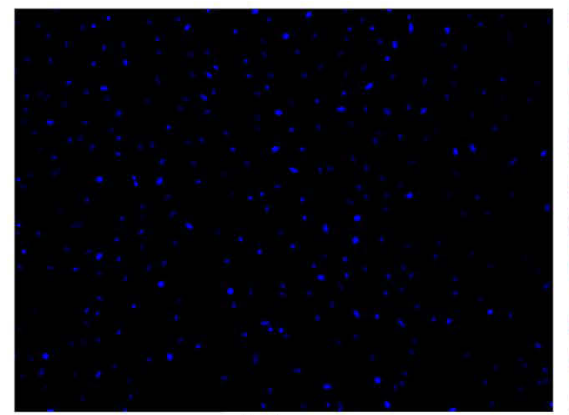

A1

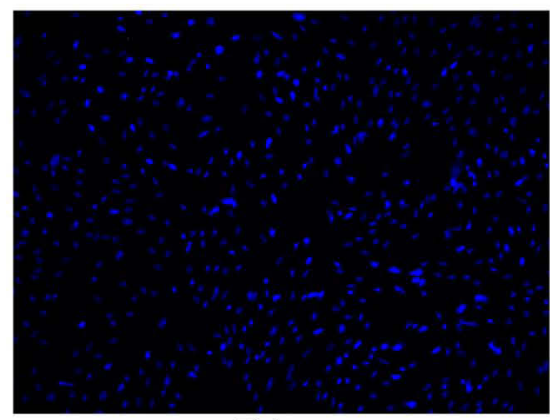

B1

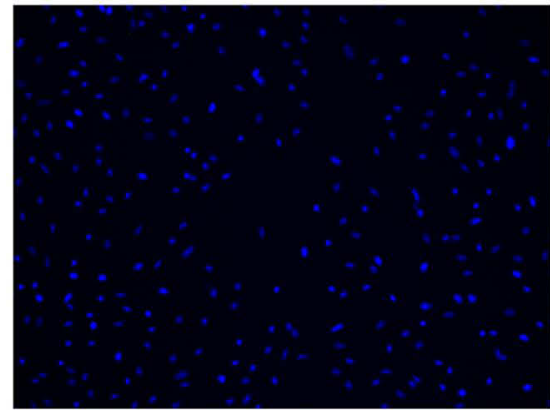

C1

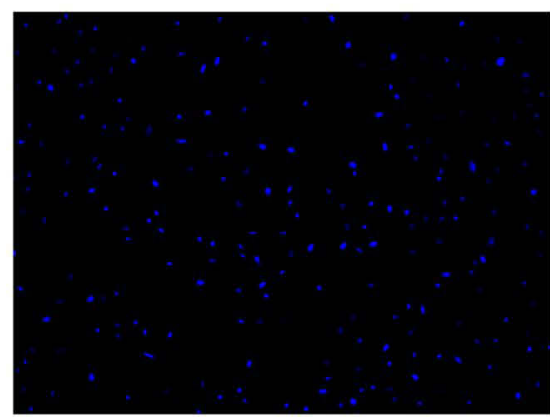

D1

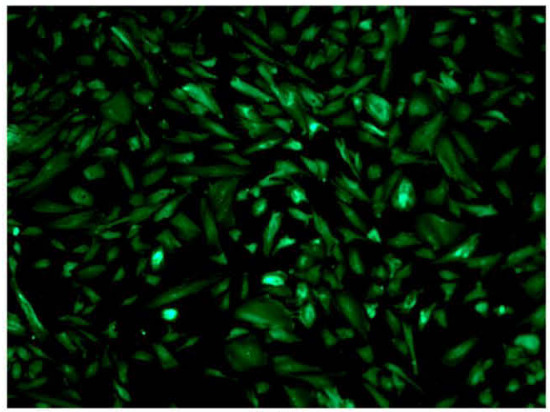

A2

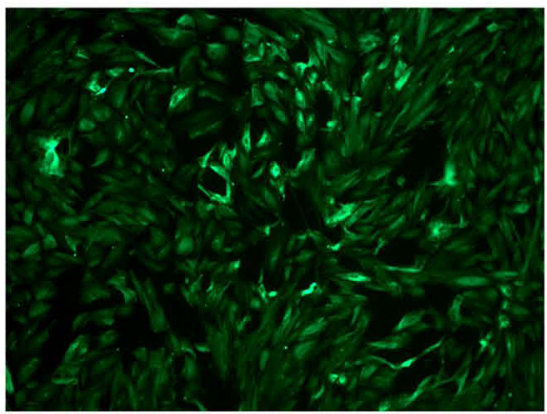

B2

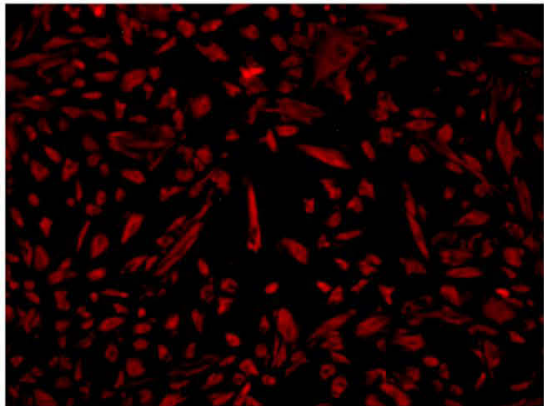

C2

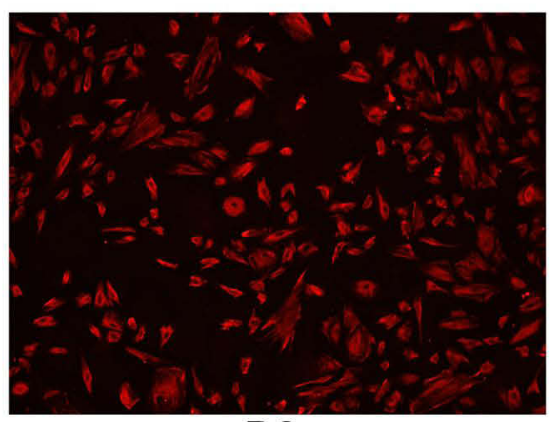

D2

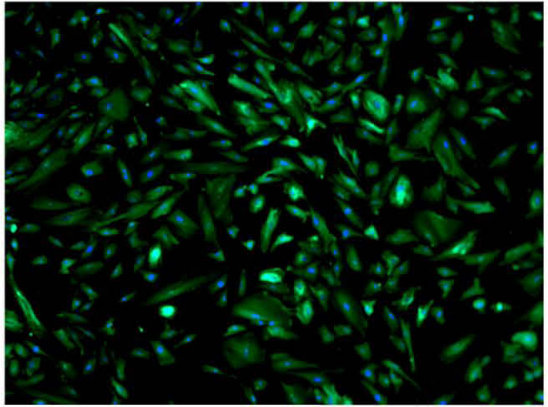

A3

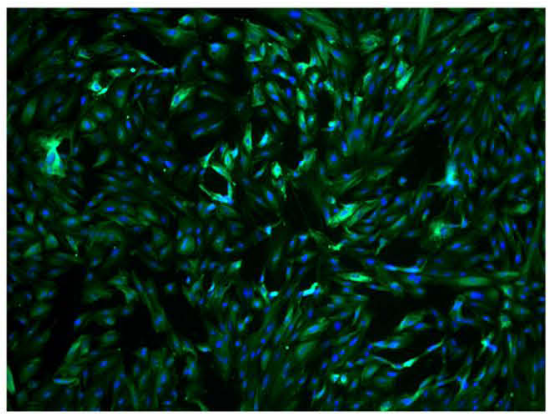

B3

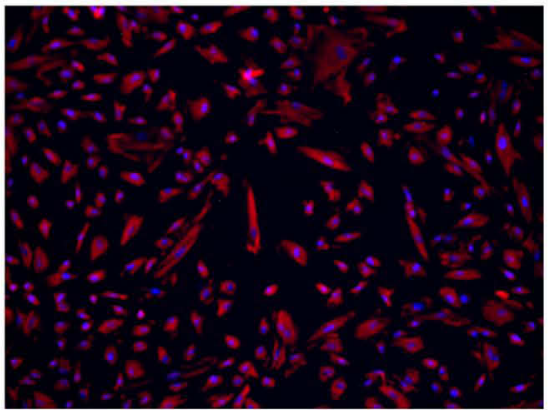

C3

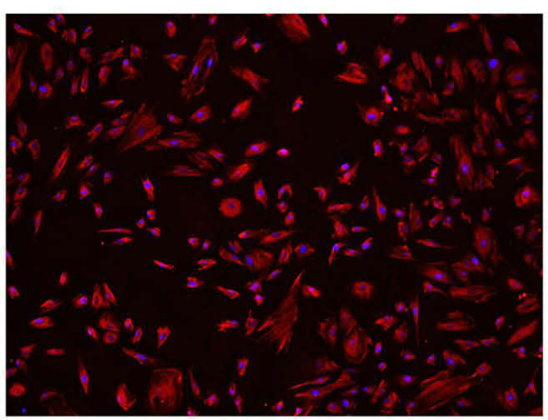

D3

Figure 2 Immunofluorescence identification of epithelial marker keratin antibody was performed on endometrial cells and oviduct mucosa cells of the second generation. The results showed that $80-90 \%$ of the cytoplasm of endometrial cells and oviduct mucosa cells were stained with green fluorescence, showing positive cytokeratin antibodies. Microscopically, the cells were polygonal, spindle and spindle. Immunofluorescence identification of interstitial cell marker vimentin antibody was performed on endometrial cells and oviduct mucosa cells of the fifth generation. $80 \%$ of the cytoplasm of endometrial cells and oviduct mucosa cells were stained with red fluorescence under microscope. It showed positive cell vimentin antibody, and the cells were fusiform and polygonal under the microscope. (AI) The second generation of endometrial cells (DAPI). (A2) The second generation of endometrial cells (CK19). (A3) The second generation of endometrial cells (Merge). (BI) The second generation of oviduct mucosal cells (DAPI). (B2) The second generation of oviduct mucosal cells (CK19). (B3) The second generation of oviduct mucosal cells (Merge). (CI) The fifth generation of endometrial cells (DAPI). (C2) The fifth generation of endometrial cells (Vimentin). (C3) The fifth generation of endometrial cells (Merge). (DI) The fifth generation of oviduct mucosal cells (DAPI). (D2) The fifth generation of oviduct mucosal cells (Vimentin). (D3) The fifth generation of oviduct mucosal cells (Merge). 


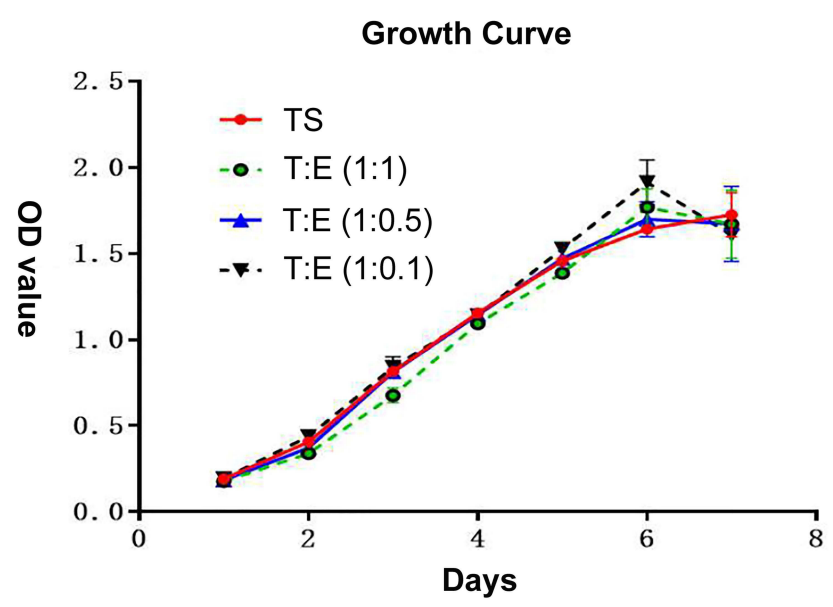

Figure 3 According to the same grouping method as mentioned above, the growth curve was drawn after 7 days of continuous measurement of endometrial cells and oviduct mucosa cells after co-culture. It can be seen that the growth rate of oviduct mucosa cells when growing alone was similar to that of endometrial cells in different proportions when co-culture, without significantly statistical difference $(P>0.05)$. The growth curve is of "S" type, which means that all of them experienced three stages: slow growth stage, logarithmic growth stage and plateau stage. TS: oviduct mucosal cells were cultured alone; $T: E(I: I)$ : the proportion of oviduct mucosal cells and endometrial cells was $I: I ; T: E(I: 0.5)$ : the proportion of oviduct mucosal cells and endometrial cells was $1: 0.5 ; \mathrm{T}: \mathrm{E}(\mathrm{I}: 0 . \mathrm{I})$ : the proportion of oviduct mucosal cells and endometrial cells was $1: 0.1$.

and all of them were fusiform, spindle, and polygonal, a round, large and centered cell nucleus (Figure 5).

\section{Discussion}

Embryo implantation requires two crucial conditions. The first is the blastocyst formation, and the second is the endometrium, which has embryo receptivity. The implantation requires maturation of the endometrium with a certain thickness and endometrium epithelial cells and decidualization of stromal cells. The differentiation and maturation of the endometrium are known as the "implantation window" allowing embryo implantation. The synchronization of the two is vital. Studies have shown that embryo quality only accounts for one-third of implantation failure, and the other two-thirds are due to poor receptivity of the endometrium. ${ }^{9}$ Endometrium deletion and thin endometrium are important causes of poor endometrium receptivity, and therefore one of the key factors affecting the success of IVF-ET. Thin endometrium refers to the thickness of the endometrium that cannot reach the embryo implantation threshold. At present, it is generally believed that the minimum thickness of the endometrium during embryo implantation should be $7 \mathrm{~mm}$, preferably greater than $9 \mathrm{~mm}$, but not more than $14 \mathrm{~mm}$. The endometrium thickness between 9-14 $\mathrm{mm}$ had the highest pregnancy rate. ${ }^{10-12}$ However, there is no suitable method to solve the problem of thin endometrium and endometrium deficiency.
The etiology of IUA is due to the destruction of the endometrium basal layer due to various factors, lack of a source of endometrium regenerated cells, uterine wall fibrosis, uncontrolled deposition of extracellular matrix (ECM) and fibrous collagen, and replacement of the interstitial septum by fibrous tissue. The glands are replaced by inactive square columnar epithelium, so there is not enough endometrium to cover the uterine cavity, leading to intrauterine adhesions.

Due to the lack of endometrium regenerated cells in the uterus after extremely severe intrauterine adhesion separation surgery, endometrium could not grow even if given several drugs, resulting in a recurrence of IUA. Therefore, to prevent re-adhesion after IUA separation surgery, new cells need to be provided to the uterine cavity to grow and cover the uterine wall, and the new endometrium tissue should have the function of the endometrium. Mesenchymal stem cells (MSCs) are a kind of pluripotent stem cell, which have all the commonalities of stem cells, namely the ability of self-renewal and multidirectional differentiation. They are a research hotspot in regenerative medicine. Multiple sources of MSCs have been used for endometrium deficiency and thin endometrium studies, from animal models to human clinical trials, such as bone marrow, fat, menstrual blood, endometrium, umbilical cord, and oviduct. It has shown promising effects in promoting endometrium growth, improving endometrium basal layer regeneration capacity, inhibiting fibrosis, promoting angiogenesis, improving endometrium receptivity, and immune regulation, thus, improving patients' reproductive outcomes. ${ }^{13-19}$

However, it was found in a long-term follow-up that the clinical application of stem cells has caused many ethical and safety issues. For example, the local microenvironment of the transplanted site of MSCs cannot limit the differentiation of MSCs, and the uncertainty of its long-term differentiation may lead to unwanted differentiation in vivo, leading to serious consequences of exacerbation of the disease. In addition, MSCs may also promote tumor growth and metastasis by building a bridge between anti-tumor immune response and neovascularization in malignant tumors. Due to its immunomodulatory properties, it inhibits anti-tumor immune response, leading to increased tumor growth. ${ }^{20-29}$ Its uncertain direction of differentiation, outcome, and malignant potential are significant safety issues, leading to doubts in the medical community regarding its therapeutic potential in the field of regenerative medicine. ${ }^{30}$ Therefore, better alternatives are needed.

Based on the similarity and homology between oviduct mucosa and endometrium mentioned above, we speculated that 


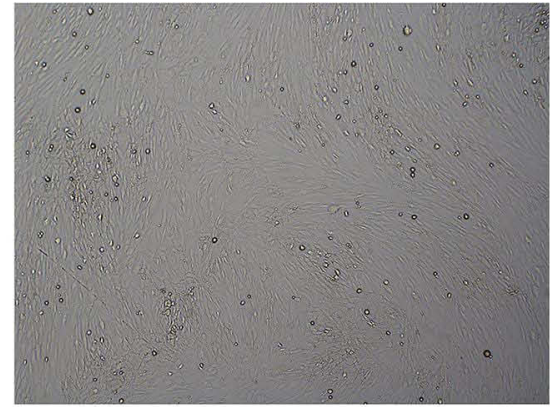

A1

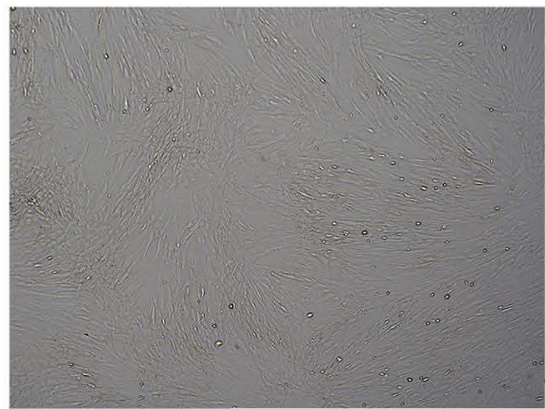

B1

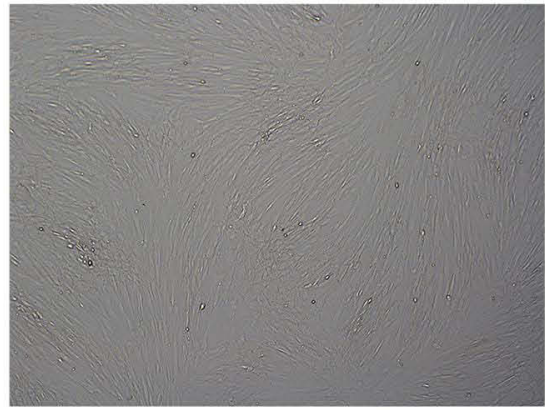

C1

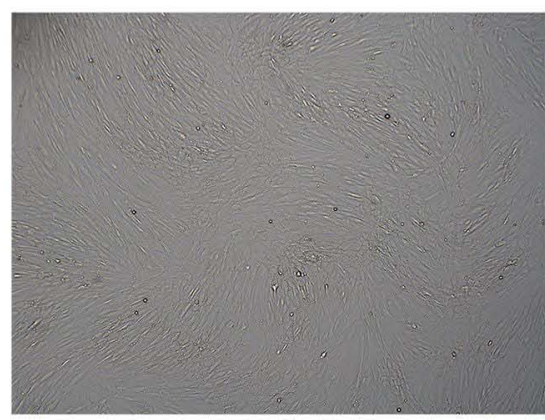

D1

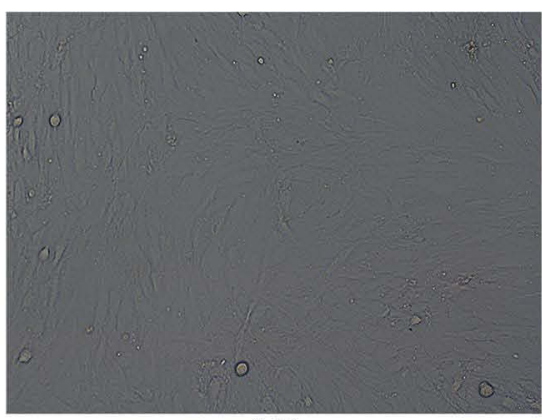

A2

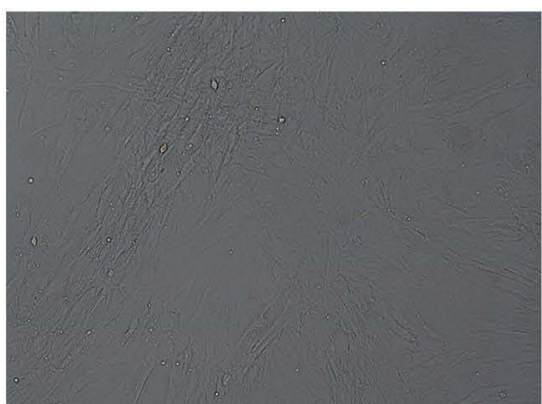

B2

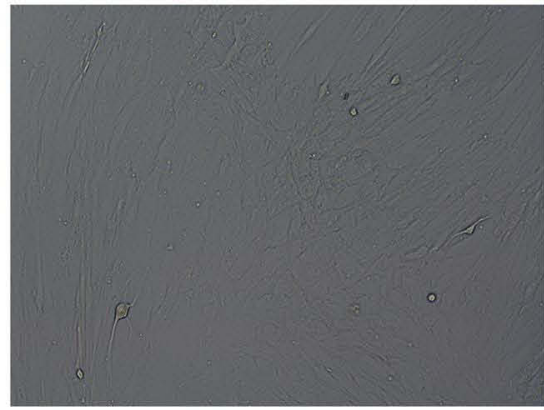

C2

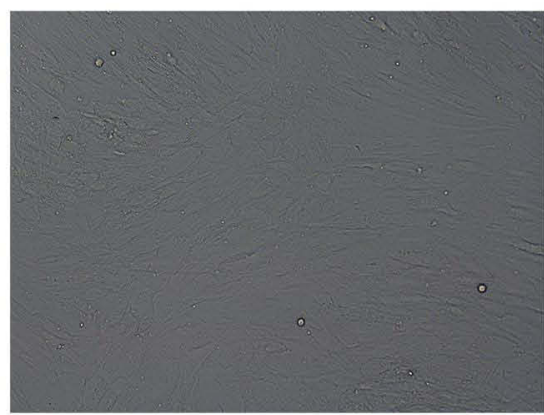

D2

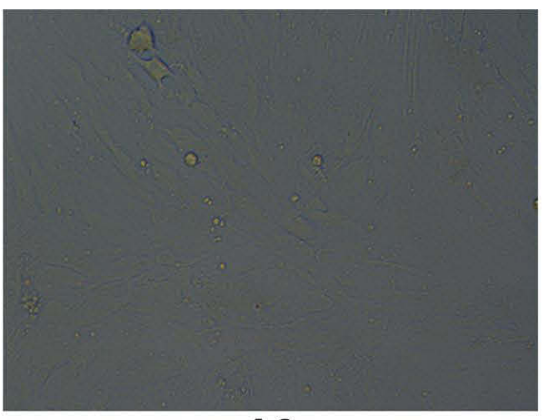

A3

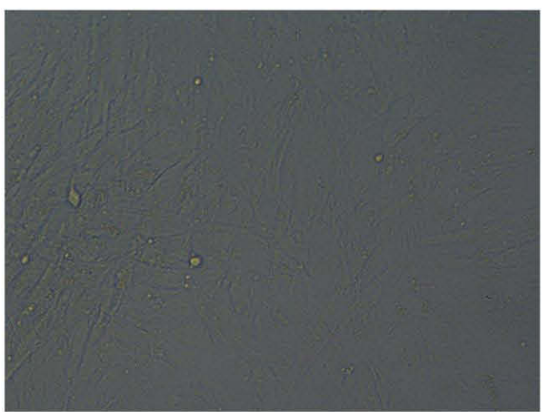

B3

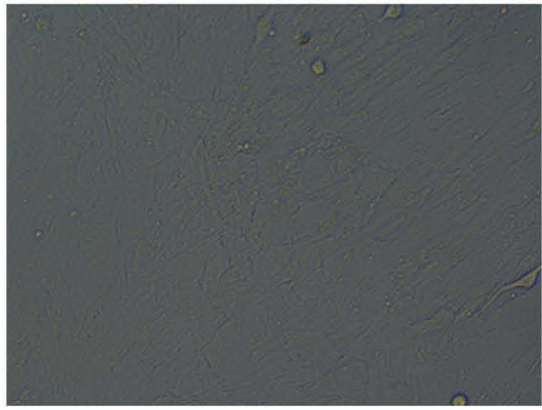

C3

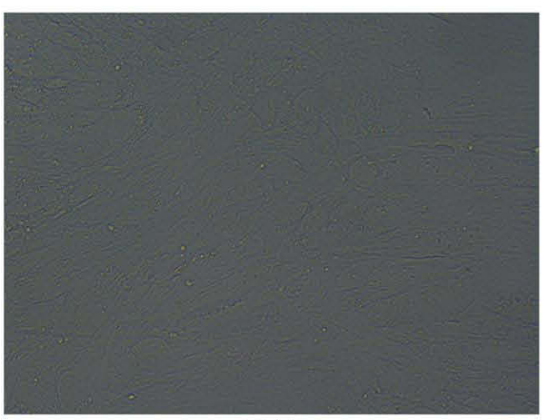

D3

Figure 4 According to the same grouping method as mentioned above, endometrial cells were cocultured with oviduct mucosa cells and treated with decidualization. After 48 hours of culture, oviduct mucosa cells induced by decidualization began to show decidual-like changes, with large and round cell volumes, rich and transparent cytoplasm, and blurred cell boundaries. (AI) TS(40x), (A2) TS(I00x), (A3) TS(200x), (BI) T: E(I:I)(40x), (B2) T:E(I:I)(I00x), (B3) T;E(I:I)(200x), (CI) T:E(I:0.5)(40x), (C2) T:E (I:0.5)(I00x), (C3) T:E(I:0.5)(200x), (DI) T:E(I:0.I)(40x), (D2) T:E(I:0.I)(I00x), (D3) T:E(I:0.I)(200x).

patients' autologous oviduct mucosa could be transplanted into the uterine cavity to replace endometrium. In order to understand whether oviduct mucosa can grow in the uterine cavity and its growth situation, we used the culture fluid of endometrial cells cultured in vitro as the simulated endometrium microenvironment and put it into oviduct mucosa cells. In order to understand the coexistence of these two types of cells in the same environment, we observed the growth of oviduct mucosa cells in 


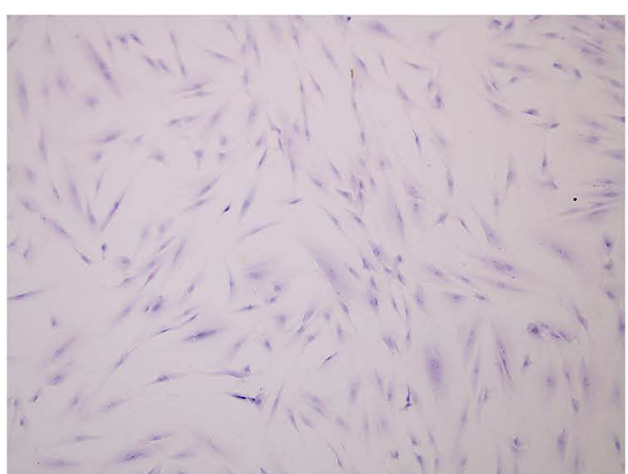

A1

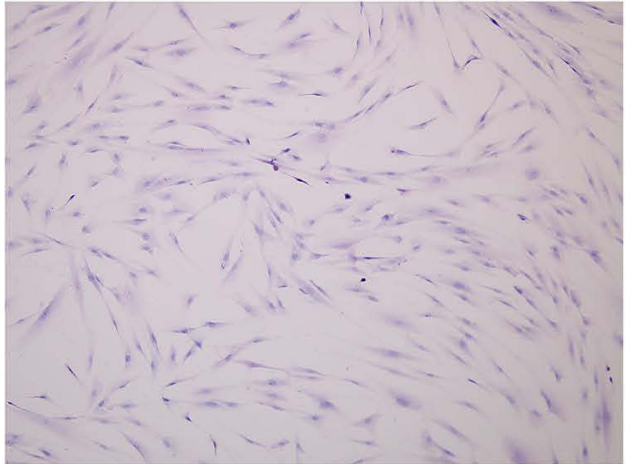

B1

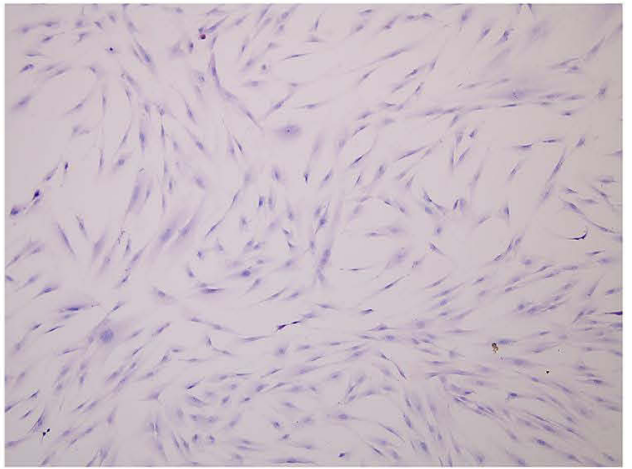

C1

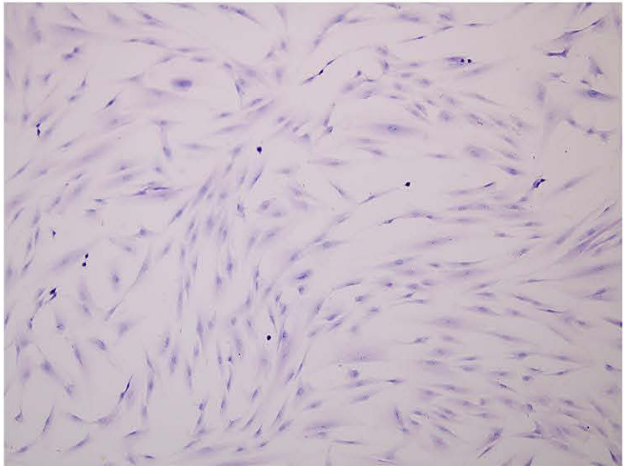

D1

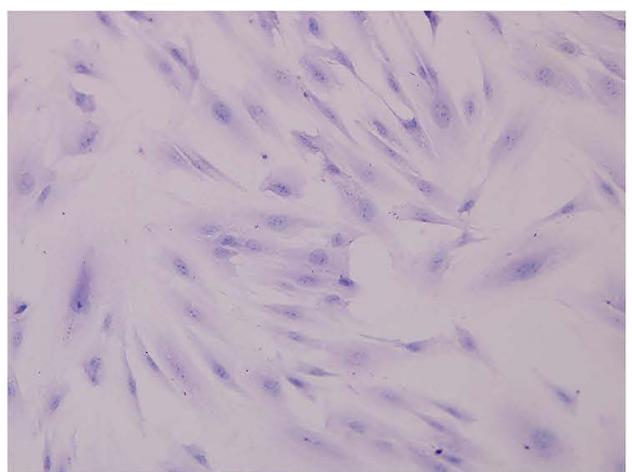

A2

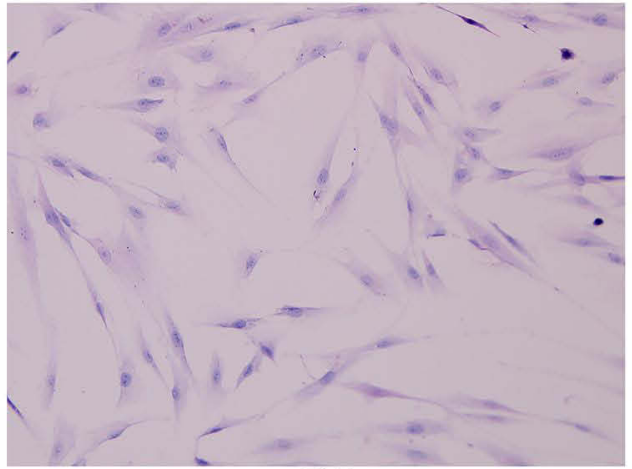

B2

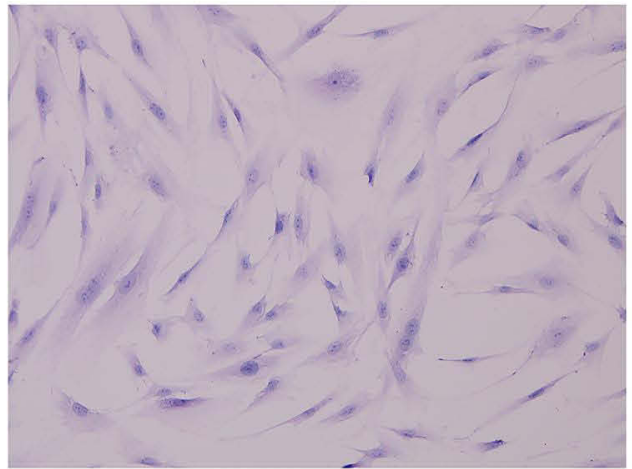

C2

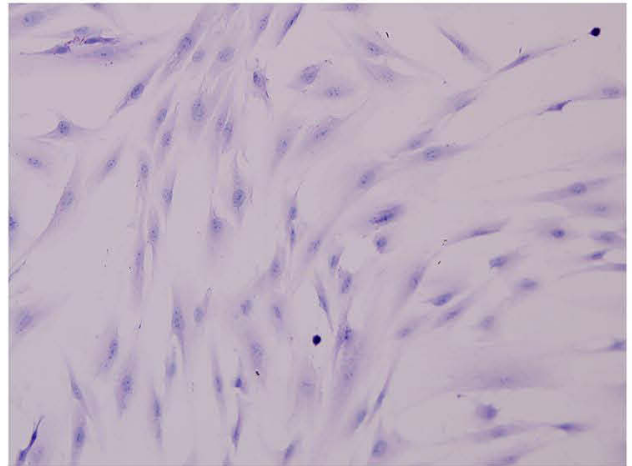

D2

Figure 5 The cells in each group were treated with decidualization by HE staining, and the cytoplasm was stained eosin and the nucleus was blue. When oviduct mucosa cells were cultured alone and co-cultured with endometrial cells of different proportions, the cell morphology was basically unchanged, and all of them were fusiform, spindle and polygonal, a round, large and centered cell nucleus. (AI) TS(I00x), (A2) TS(200x), (BI) T:E(I:I)(I00x), (B2) T:E(I:I)(200x), (CI) T:E(I:0.5)(I00x), (C2) T:E(I:0.5) (200x), (DI) T:E(I:0.I)(I00x), (D2) T:E(I:0.I)(200x). 
different concentrations of endometrial cells. A CCK8 cell proliferation experiment was conducted on oviduct mucosa cells grown in four groups of endometrial cell culture solutions with different concentrations. The growth curve indicated that oviduct mucosa cells could grow in a uterine environment with different endometrial proportions. The growth rate of oviduct mucosa was similar to that in the environment without endometrial cells, without excessive proliferation, suggesting that oviduct mucosa can grow in the endometrium microenvironment without being affected by the endometrium. The growth rate of endometrial cells in the co-culture environment was also consistent with that in the individual culture, suggesting that cell growth from the two sources did not inhibit each other.

In order to further understand the changes in the morphology and structure of oviduct cells in the uterine environment, we placed oviduct mucosa cells on the bottom of the co-culture dish and allowed them to grow in different concentrations of endometrial cells, and then took them out for HE staining. The cell morphology of the co-cultured oviduct mucosa cells was observed under a microscope when they grew in the culture solution of endometrial cells of different concentrations. The cell morphology was similar to that of the single culture, showing fusiform, polygonal, and fusiform, a round, large and centered cell nucleus. These results indicated that the cell morphology of oviduct mucosa cells was not affected when co-cultured with endometrial cells. In the co-culture system, endometrial cells and oviduct mucosa both grew at the same rate as when they were grown alone. Therefore, we speculated that in living tissues, oviduct mucosa might grow in the uterine cavity or be suitable for the growth of oviduct mucosa in the uterine cavity without affecting the growth of the original endometrium.

Decidualization is a progesterone-dependent differentiation process of endometrium mesenchymal cells, which refers to the transformation of endometrium mesenchymal fibroblasts into specialized secretory decidua cells, which provide an essential nutritional and immune environment for embryo implantation and placental development. It is a necessary condition for successful embryo implantation. In order to observe whether oviduct mucosa cells can undergo decidualization after coculture and whether they have the conditions for embryo implantation, we also conducted in vitro decidualization induction after co-culture of two kinds of cells. It was found that after in vitro decidualization induction, the volume of oviduct mucosa cells became larger and rounded, the cytoplasm was rich and transparent, and the cell boundary became blurred and morphologically changed, which was the same as the cell morphology of endometrium decidualization.
In our study, we also observed that only part of the oviduct mucosa cells induced by in vitro decidualization after co-culture had morphologic changes of decidualization cells, but not all of them. This is also consistent with the study of oviduct mucosa taken from living tissues. $^{31}$

Although salpingocyesis often has a bad outcome, it is currently believed that its causes include insufficient blood supply, excessively thin oviduct mucosa and muscle layer, insufficient decidualization reaction, ${ }^{32}$ and abnormal secretion of certain related factors. Therefore, if oviduct mucosa is put into the uterine cavity as a substitute for endometrium, it can at least solve the factors of its limited anatomical structure. However, insufficient oviduct mucosa decidualization may lead to abortion, placental implantation, and other outcomes. Enhancing its decidualization is a problem that needs to be solved both in the laboratory and clinic.

In conclusion, our results indicate that oviduct mucosa cells can grow normally in the environment with endometrium, and their morphology and growth speed are not affected. Morphological changes of oviduct mucosa cells can occur after in vitro decidualization treatment in the symbiosis of the two cells. There was no significant difference between the two cells in separate culture and co-culture in terms of HE staining. These findings lay a foundation for the possibility of oviduct mucosa instead of endometrium in the treatment of IUA. However, whether the growth of cells in vitro is consistent with that in vivo requires further animal experimental studies, and our current study has not yet involved the study of the specific functions of the two cells. Therefore, these questions need to be more important before it becomes the possibility of routine clinical practice. Many further experiments will be solved one by one, such as the comparative study of the related functions of the symbiotic fallopian tube mucosal cells and normal endometrial cells during embryo implantation, immune status, etc.

\section{Ethics Approval and Consent to Participate}

The study was conducted in accordance with the Declaration of Helsinki (as was revised in 2013). The study was approved by Ethics Committee of Affiliated Hospital of Guizhou Medical University [No. 2020-(264$01)]$. Written informed consent was obtained from all participants. 


\section{Acknowledgments}

We are particularly grateful to all the people who have given us help on our article.

\section{Funding}

This study was funded by the science and technology fund project of health Committee of Guizhou Province, China (No. gzwjkj2020-1-146).

\section{Disclosure}

The authors declare that they have no competing interests.

\section{References}

1. Senturk LM, Erel CT. Thin endometrium in assisted reproductive technology. Curr Opin Obstet Gynecol. 2008;20(3):221-228. doi:10.1097/GCO.0b013e328302143c

2. Varga I, Miko M, Kachlík D, Žišková M, Danihel L' Jr, Babál P. How many cell types form the epithelial lining of the human uterine tubes? Revision of the histological nomenclature of the human tubal epithelium. Ann Anat. 2019;224:73-80.

3. Saint-Dizier M, Schoen J, Chen S, Banliat C, Mermillod P. Composing the early embryonic microenvironment: physiology and regulation of oviductal secretions. Int J Mol Sci. 2019;21(1):223.

4. Leese HJ. The formation and function of oviduct fluid. $J$ Reprod Fertil. 1988;82(2):843-856.

5. Makrigiannakis A, Karamouti M, Petsas G, Makris N, Nikas G, Antsaklis A. The expression of receptivity markers in the fallopian tube epithelium. Histochem Cell Biol. 2009;132(2):159-167.

6. Qin L, Wang YL. Molecular basis of tubal pregnancy implantation window and expression of maternal-fetal interface related molecules. J Reprod Med. 2003;06:369-373.

7. Sülz L, Valenzuela JP, Salvatierra AM, Ortiz ME, Croxatto HB. The expression of alpha(v) and beta3 integrin subunits in the normal human Fallopian tube epithelium suggests the occurrence of a tubal implantation window. Hum Reprod. 1998;13(10):2916-2920.

8. Goddijn M, van der Veen F, Schuring-Blom GH, Ankum WM, Leschot NJ. Cytogenetic characteristics of ectopic pregnancy. Hum Reprod. 1996;11(12):2769-2771.

9. Craciunas L, Gallos I, Chu J, et al. Conventional and modern markers of endometrial receptivity: a systematic review and meta-analysis. Hum Reprod Update. 2019;25(2):202-223.

10. Momeni M, Rahbar MH, Kovanci E. A meta-analysis of the relationship between endometrial thickness and outcome of in vitro fertilization cycles. J Hum Reprod Sci. 2011;4(3):130-137.

11. Sharma S, Rani G, Bose G, Saha I, Bathwal S, Chakravarty BN. Tamoxifen is Better than Low-Dose Clomiphene or Gonadotropins in Women with Thin Endometrium $(<7 \mathrm{~mm})$ after clomiphene in intrauterine insemination cycles: a prospective study. J Hum Reprod Sci. 2018;11 (1):34-39.

12. Zadehmodarres S, Salehpour S, Saharkhiz N, Nazari L. Treatment of thin endometrium with autologous platelet-rich plasma: a pilot study. JBRA Assist Reprod. 2017;21(1):54-56.

13. Nagori CB, Panchal SY, Patel H. Endometrial regeneration using autologous adult stem cells followed by conception by in vitro fertilization in a patient of severe Asherman's syndrome. J Hum Reprod Sci. 2011;4(1):43-48.
14. Gao L, Huang Z, Lin H, Tian Y, Li P, Lin S. Bone Marrow Mesenchymal Stem Cells (BMSCs) restore functional endometrium in the rat model for severe Asherman syndrome. Reprod Sci. 2019;26 (3):436-444.

15. Wang J, Ju B, Pan C, et al. Application of bone marrow-derived mesenchymal stem cells in the treatment of intrauterine adhesions in rats. Cell Physiol Biochem. 2016;39(4):1553-1560.

16. Santamaria X, Cabanillas S, Cervelló I, et al. Autologous cell therapy with $\mathrm{CD} 133+$ bone marrow-derived stem cells for refractory Asherman's syndrome and endometrial atrophy: a pilot cohort study. Hum Reprod. 2016;31(5):1087-1096.

17. Zhao G, Cao Y, Zhu X, et al. Transplantation of collagen scaffold with autologous bone marrow mononuclear cells promotes functional endometrium reconstruction via downregulating $\Delta \mathrm{Np} 63$ expression in Asherman's syndrome. Sci China Life Sci. 2017;60(4):404-416.

18. Tan J, Li P, Wang Q, et al. Autologous menstrual blood-derived stromal cells transplantation for severe Asherman's syndrome. Hum Reprod. 2016;31(12):2723-2729.

19. Cao Y, Sun H, Zhu H, et al. Allogeneic cell therapy using umbilical cord MSCs on collagen scaffolds for patients with recurrent uterine adhesion: a Phase I clinical trial. Stem Cell Res Ther. 2018;9(1):192.

20. Breitbach M, Bostani T, Roell W, et al. Potential risks of bone marrow cell transplantation into infarcted hearts. Blood. 2007;110 (4):1362-1369.

21. Yoon YS, Park JS, Tkebuchava T, Luedeman C, Losordo DW. Unexpected severe calcification after transplantation of bone marrow cells in acute myocardial infarction. Circulation. 2004;109 (25):3154-3157.

22. Kuriyan AE, Albini TA, Townsend JH, et al. Vision loss after intravitreal injection of autologous "Stem cells" for AMD. $N$ Engl J Med. 2017;376(11):1047-1053.

23. Lazennec G, Jorgensen C. Concise review: adult multipotent stromal cells and cancer: risk or benefit? Stem Cells. 2008;26(6):1387-1394.

24. Patel SA, Meyer JR, Greco SJ, Corcoran KE, Bryan M, Rameshwar P. Mesenchymal stem cells protect breast cancer cells through regulatory $\mathrm{T}$ cells: role of mesenchymal stem cell-derived TGF-beta. J Immunol. 2010;184(10):5885-5894.

25. Wang X, Zhang Z, Yao C. Survivin is upregulated in myeloma cell lines cocultured with mesenchymal stem cells. Leuk Res. 2010;34 (10):1325-1329.

26. Ljujic B, Milovanovic M, Volarevic V, et al. Human mesenchymal stem cells creating an immunosuppressive environment and promote breast cancer in mice. Sci Rep. 2013;3:2298.

27. Oswald J, Boxberger S, Jørgensen B, et al. Mesenchymal stem cells can be differentiated into endothelial cells in vitro. Stem Cells. 2004;22(3):377-384.

28. Janeczek Portalska K, Leferink A, Groen N, et al. Endothelial differentiation of mesenchymal stromal cells. PLoS One. 2012;7(10): e46842.

29. Khakoo AY, Pati S, Anderson SA, et al. Human mesenchymal stem cells exert potent antitumorigenic effects in a model of Kaposi's sarcoma. J Exp Med. 2006;203(5):1235-1247.

30. Volarevic V, Ljujic B, Stojkovic P, Lukic A, Arsenijevic N, Stojkovic M. Human stem cell research and regenerative medicinepresent and future. Br Med Bull. 2011;99:155-168.

31. Randall S, Buckley CH, Fox H. Placentation in the fallopian tube. Int J Gynecol Pathol. 1987;6(2):132-139.

32. Floridon C, Nielsen O, Holund B, et al. Localization and significance of urokinase plasminogen activator and its receptor in placental tissue from intrauterine, ectopic and molar pregnancies. Placenta. 1999;20 (8):711-721. 


\section{Publish your work in this journal}

The International Journal of Women's Health is an international, peerreviewed open-access journal publishing original research, reports, editorials, reviews and commentaries on all aspects of women's healthcare including gynecology, obstetrics, and breast cancer. The manuscript management system is completely online and includes a very quick and fair peer-review system, which is all easy to use. Visit http://www.dovepress.com/testimonials.php to read real quotes from published authors.

Submit your manuscript here: https://www.dovepress.com/international-journal-of-womens-health-journal 